\title{
Defence transcriptome assembly and pathogenesis related gene family analysis in Pinus tecunumanii (low elevation)
}

\author{
Erik A. Visser', Jill L. Wegrzyn², Alexander A. Myburg ${ }^{1}$ and Sanushka Naidoo ${ }^{1 *}$
}

\begin{abstract}
Background: Fusarium circinatum is a pressing threat to the cultivation of many economically important pine tree species. Efforts to develop effective disease management strategies can be aided by investigating the molecular mechanisms involved in the host-pathogen interaction between F. circinatum and pine species. Pinus tecunumanii and Pinus patula are two closely related tropical pine species that differ widely in their resistance to $F$. circinatum challenge, being resistant and susceptible respectively, providing the potential for a useful pathosystem to investigate the molecular responses underlying resistance to $F$. circinatum. However, no genomic resources are available for $P$. tecunumanii. Pathogenesis-related proteins are classes of proteins that play important roles in plant-microbe interactions, e.g. chitinases; proteins that break down the major structural component of fungal cell walls. Generating a reference sequence for $P$. tecunumanii and characterizing pathogenesis related gene families in these two pine species is an important step towards unravelling the pine- $F$. circinatum interaction.

Results: Eight reference based and 12 de novo assembled transcriptomes were produced, for juvenile shoot tissue from both species. EvidentialGene pipeline redundancy reduction, expression filtering, protein clustering and taxonomic filtering produced a $50 \mathrm{Mb}$ shoot transcriptome consisting of 28,621 contigs for $P$. tecunumanii and a $72 \mathrm{Mb}$ shoot transcriptome consisting of 52,735 contigs for $P$. patula. Predicted protein sequences encoded by the assembled transcriptomes were clustered with reference proteomes from 92 other species to identify pathogenesis related gene families in P. patula, P. tecunumanii and other pine species.

Conclusions: The $P$. tecunumanii transcriptome is the first gene catalogue for the species, representing an important resource for studying resistance to the pitch canker pathogen, F. circinatum. This study also constitutes, to our knowledge, the largest index of gymnosperm PR-genes to date.
\end{abstract}

Keywords: Pinus tecunumanii, Pinus patula, Transcriptome assembly, PR genes, Fusarium circinatum, Disease resistance

\section{Background}

The pitch canker fungus Fusarium circinatum Nirenberg and O'Donnell [1] has resulted in losses in pine plantations, seed orchards and nurseries worldwide [2, 3]. With a host range of more than 60 Pinus spp. [2], many of which are commercially important, this pathogen poses a significant threat to both forestry and conservation. The wide range of inter- and intraspecific variation

\footnotetext{
* Correspondence: sanushka.naidoo@fabi.up.ac.za

${ }^{1}$ Department of Biochemistry, Genetics and Microbiology, Forestry and

Agricultural Biotechnology Institute (FABI), Genomics Research Institute (GRI),

University of Pretoria, Private bag X20, Pretoria 0028, South Africa

Full list of author information is available at the end of the article
}

in susceptibility of Pinus spp. to F. circinatum [4, 5] holds the potential for effective disease management through genetic resistance.

Development of more resistant families and genotypes for susceptible Pinus spp. [6] as well as selection and generation of resistant hybrids [7] have shown promise for long-term management of $F$. circinatum. Breeding and selection approaches, however, are time consuming and use of resistant genotypes could select for novel pathotypes [2]. Knowledge of the molecular mechanisms underlying resistance could expedite development of resistant genotypes and improve the effectiveness of genetic resistance.

(c) The Author(s). 2018 Open Access This article is distributed under the terms of the Creative Commons Attribution 4.0 International License (http://creativecommons.org/licenses/by/4.0/), which permits unrestricted use, distribution, and 
The majority of information related to plant-pathogen interactions originate from studies on model plant species such as Arabidopsis thaliana and Nicotiana spp. [8]. Comparatively few studies have investigated these interactions in trees, and even less in gymnosperms. The main barrier to studying defence responses in non-model organisms in the past was the need for a reference genome. Among plants, particularly gymnosperms, genome size and complexity hindered sequencing and assembly of a reference genome. The availability of next-generation sequencing technology has circumvented this barrier for non-model organisms by enabling transcriptome assembly from high-throughput RNA sequencing (RNA-seq) data $[9,10]$.

While recent transcriptomic studies investigated the Pinus-F. circinatum interaction $[11,12]$, both studies focussed on susceptible species. Knowledge of defence mechanisms in the resistant interaction is lacking. The low elevation provenance (LE) of Tecun Uman Pine $(P$. tecunumanii Eguiluz \& J. P. Perry) is an economically important F. circinatum resistant [4] Pinus species that has shown promise in hybridisation trials with $P$. patula, making it a good candidate for use as a model of resistance.

Studies on model plants have shown that molecular defence mechanisms consist of complex, multi-level processes [8]. In short, pathogen recognition occurs either through membrane binding of bound recognition receptors (PRRs) to pathogen-/damage-/microbe-associated molecular patterns (PAMPs, DAMPs, MAMPs) or through interaction between host resistance (R) genes and pathogen secreted effector proteins [13-15]. Pathogen perception results in activation of signal transduction cascades that initiate various local and systemic host defence responses including: reactive oxygen species (ROS) generation, cell wall modification, phytohormone defence pathways, defence-related protein expression and induction of systemic acquired resistance (SAR) [16-21]. An important group of markers for SAR are the pathogenesis-related (PR) proteins, a group of proteins identified due to their induction during biotic stress and direct antimicrobial activity of some PR gene family members [21-23]. There are currently 17 described PR gene families, classified by amino acid sequence and enzymatic activity, numbered in order of their description [22, 23]. Despite being the first family identified, a potential mode of action for the PR-1 proteins in plant defence was only recently described [24]. PR-1 proteins were shown to bind and sequester sterols, directly inhibiting sterol-auxotrophic pathogens as well as sterol-prototrophic pathogens with compromised sterol biosynthesis [24]. The PR-9 family are peroxidases that could be involved in cell wall reinforcement through catalysing lignification [25]. This could result in enhanced resistance against multiple pathogens [22, 23]. There are also two putative novel PR gene families, the PR-18 carbohydrate oxidases identified from Helianthus annuus and
Lactuca sativa [26], and the PR-19 anti-microbial peptides identified from Macadamia integrifolia and Pinus sylvestris $[27,28]$.

This study aimed to produce a reference sequence for $P$. tecunumanii transcriptome profiling and a comparable reference for $P$. patula, as a resource for further investigation of the Pinus-F. circinatum host pathogen interaction, and to use the generated resources to identify the PR-gene families within these species. High quality reference transcriptomes were assembled, including the first gene catalogue for $P$. tecunumanii to date. These references were used to identify 639 and 785 PR gene candidates in $P$. tecunumanii and $P$. patula respectively.

\section{Methods}

Plant material and inoculation trial

Four month old low elevation (LE) Pinus tecunumanii seedlings, representing four open pollinated families, were sourced from SAPPI (Shaw Research Centre, South Africa) and 4 month old $P$. patula seedlings from a single open pollinated family were sourced from Mondi Forests (Trahar Technology Centre, South Africa). Seedlings were maintained in the Forestry and Agricultural Biotechnology Institute (FABI) Fusarium screening greenhouse at the University of Pretoria experimental unit. Pathogen challenge was performed as described in Visser et al. [12]. Briefly, seedlings were inoculated with $F$. circinatum isolate FCC3579, harvested from cultures on $1 / 2$ strength potato dextrose agar ( $1 / 2$ PDA; Merck) washed with $15 \%(v / \mathrm{v})$ sterile glycerol and diluted to $5 \times 10^{4}$ spores $/ \mathrm{mL}$, or mock-inoculated with $15 \%(\mathrm{v} / \mathrm{v})$ sterile glycerol, at 6 months old by clipping the apical bud and depositing $10 \mu \mathrm{L}$ inoculum on the wound. Tissue was harvested from seedlings at three and 7 days post inoculation (dpi) for three biological replicates (BR) per group. The top $1 \mathrm{~cm}$ of shoot tissue, from the point of inoculation, from 16 seedlings was pooled for each biological replicate. Harvested tissue was flash frozen in liquid nitrogen and stored at $-80{ }^{\circ} \mathrm{C}$ until use. Disease progression was monitored by measuring stem length and stem discolouration from the point of inoculation over 6 weeks to calculate the percentage live stem ([stem length $(\mathrm{mm})$-stem discolouration $(\mathrm{mm})] /$ stem length $(\mathrm{mm}))$. The difference in mean percentage live stem between inoculated and mock-inoculated plants at each time point was analysed using a Kruskal-Wallis rank sum test $(p<0.05)$. Re-isolation of the pathogen was performed by placing tissue harvested at $14 \mathrm{dpi}$ on $1 / 2 \mathrm{PDA}$ and observing culture morphology after 7 days.

\section{RNA isolation and sequencing}

Total RNA was extracted from homogenised samples, ground in a mortar and pestle, using the Plant/Fungi RNA Purification Kit (Norgen Biotek Corp., Thorold, 
ON, Canada) according to the manufacturer's instructions, with the inclusion of acid washed glass beads during lysis to improve cell break down. Extracted samples were assessed using a Bio-Rad Experion ${ }^{\mathrm{Tx}}$ automated electrophoresis system (Bio-Rad Laboratories, Hercules, CA, USA) to determine sample concentration as well as ensure sample integrity (RNA Integrity Number $>7.0$ ) and purity (absence of genomic DNA).

Inoculated and mock-inoculated samples, for both time points, were sent to Novogene (Novogene Corporation Inc., Chula Vista, CA, USA) for strand specific RNA-Sequencing on an Illumina HiSeq2500 (Illumina, San Diego, CA, USA). Samples were sequenced in three sets for each species, to optimise read length for transcriptome assembly (Additional file 1: Table S1). Sample set one consisted of a single sample of pooled RNA from all six three dpi samples (500 bp insert, PE250 sequencing). Sample set two consisted of the six samples (3 BR inoculated, 3 BR mock-inoculated) for three dpi (300 bp insert, PE125 sequencing). Sample set three consisted of the six samples (3 BR inoculated, 3 BR mock-inoculated) for seven dpi (300 bp insert, PE150 sequencing).

\section{Transcriptome assembly and annotation}

RNA-sequencing libraries obtained from Novogene were assessed using FastQC [29]. Preliminary transcriptome assembly was performed using Trinity v2.2.0 [30], on two datasets (Additional file 1: Table S1). Reads were quality trimmed and filtered using trimmomatic v0.32 (Additional file 1: Table S2) [31]. For each species, all 13 trimmed and filtered libraries were combined to produce a full dataset. In silico read normalisation, to a maximum coverage of 100 , was performed using Trinity on the full dataset to produce a normalised dataset. Both datasets were used for transcriptome assembly. Twenty strand specific preliminary assemblies were produced, with a minimum contig length of 350, using two different: $k$-mer sizes ( $25 \& 31$ ), stringencies for de Bruijn graph construction, and algorithms for transcript reconstruction (Additional file 1: Table S2). The normalised dataset for both species was mapped against the $P$. taeda v1.01 draft genome assembly using GSNAP 2016-11-07 (Genomic Short Read Alignment Program; Additional file 1: Table S2) [32] for genome guided assembly. Preliminary assemblies were filtered to obtain the longest isoform per locus, an assembly code (Additional file 1: Table S2) added to the transcript identifiers, and combined to form a highly redundant assembly. Redundancy was reduced using the EvidentialGene [33] tr2aacds pipeline v2016.07.11. Assembly statistics were calculated using Transrate v1.0.3 [34].

Primary transcripts from the tr2aacds pipeline were annotated using the eukaryotic non-model transcriptome annotation pipeline v0.7.3.2 (EnTAP) [35]. In short, the normalised dataset was mapped to the transcripts using Bowtie2 v2.3.0 [36]. Transcript expression was calculated using RSEM v1.3.0 (RNA-Seq by Expectation-Maximum) [37] and transcripts with an FPKM $<1$ (Fragments Per Kilobase of transcript per Million mapped reads) were discarded. Frame prediction was performed on transcripts with detectable expression using GeneMarkS-T v5.1 March 2014 [38], transcripts without a predicted reading frame were discarded, and predicted proteins clustered to $90 \%$ identity using Usearch v9.0.2132 [39]. Diamond v0.8.31 [40] was used to obtain protein BLAST alignments for predicted protein sequences of the remaining transcripts against: the RefSeq Complete Protein database, the UniProt-KB/Swissprot database, and the Arabidopsis thaliana proteome; using a minimum query coverage of $80 \%$, a minimum target coverage of $60 \%$ and a minimum e-value of 1e-4. Only the best hits across all three databases were retained. Functional annotation, Gene Ontology (GO) annotation relative to the full GO database and orthologous group assignment, was performed using EggNOG 4.5 [41]. Unannotated sequences were discarded as erroneously assembled transcripts. Non-pine origin sequences were removed based on best hit taxonomy (discarding sequences with best hits from archaea, fungi, insects, bacteria, viruses and vertebrates), for sequences with BLASTp alignments, and orthologous group taxonomic scope (discarding sequences not associated with Viridiplantae, Eukaryota or Ancestor lineages), for sequences without BLASTp alignments, to produce the $P$. tecunumanii (Pnte_v1.0) and P. patula (Pipt_v2.0) draft transcriptome assemblies. For ease of comparison, GO terms were normalised to level two of the classification tree. Putative TAIR10 identifiers were assigned to transcripts based on best hits to the $A$. thaliana proteome. Completeness and contiguity of the assembly was determined using BUSCO v3.0 (Benchmarking of SingleCopy Orthologs; [42]) against the eukaryote_odb9 (303 BUSCOs) and embryophyta_odb9 (1440 BUSCOs) lineages and compared to 26 other gymnosperm assemblies (Additional file 1: Table S3) obtained from the TreeGenes database [43].

\section{Orthogroup identification}

Orthologous gene clusters (orthogroups) were identified using OrthoFinder v1.1.10 [44] across 94 different species (Additional file 1: Table S4), using default settings, similar to previous studies $[45,46]$. The dataset consisted of proteomes for: the red algae Cyanidioschyzon merolae as outgroup, 11 species of green algae, the liverwort Marchantia polymorpha, the moss Physcomitrella patens, the clubmoss Selaginella moellendorffii, 14 gymnosperm species (including Pnte_v1.0 and Pipt_v2.0), the basal angiosperm Amborella trichopoda, 18 monocot species and 46 dicot species, containing a total of $2,974,043$ protein sequences. The proteomes for P. taeda, P. lambertiana and Pseudotsuga 
menziessii were obtained from the TreeGenes database [43]. All remaining proteomes were retrieved from the PLAZA database. Orthogroups containing previously identified putative PR-genes for Arabidopsis thaliana, Brachypodium distachyon, Oryza sativa, Populus trichocarpa and Vitis vinifera [47] were classified as pathogenesis-related gene families for PR-1 through PR-17. Putative PR-18 and PR-19 orthogroups were identified through BLASTp alignment of type sequences to the OrthoFinder input dataset. Both $H$. annuus and $L$. sativa carbohydrate oxidase protein sequences (Genbank accessions AAL77103.1 and AAL77102.1) [26] were used as type sequences for identification of putative PR-18 orthogroups. Putative PR-19 orthogroups were identified using four $P$. sylvestris antimicrobial peptide sequences (Genbank accessions AAL05052.1 to AAL05055.1) as well as the first antimicrobial peptide identified from $M$. integrifolia (UniProtKB/Swiss-Prot accession P80915.1).

\section{Results and discussion}

F. circinatum disease progression on $P$. tecunumanii and $P$. patula

Seedlings of $P$. tecunmanii and $P$. patula were inoculated with $F$. circinatum and the decline in percentage green stem monitored over the course of 6 weeks (Fig. 1). Stem discolouration, at the point of inoculation, was visible on all seedlings at 7 days post inoculation with no discernible difference in lesion colour or length between inoculated and mock-inoculated seedlings. By 14 dpi a clear difference in lesion colouration was visible between treatment groups for both species, with inoculated seedlings displaying purple lesions, and a significant difference in lesion length was observed between $P$. patula treatment groups $(p<0.05)$. Initial mortality of $P$. patula seedlings was observed at $21 \mathrm{dpi}$. By 42 dpi chlorosis prevented further measurements of $P$. patula seedlings. While a significant difference in percentage green stem was observed for $P$. tecunumanii seedlings after $14 \mathrm{dpi}$, this was due to more wound discolouration on mock-inoculated relative to inoculated seedlings and all seedling had more than $98 \%$ live stem. The difference in stem discolouration between $P$. tecunumanii and $P$. patula is therefore consistent with the classification of $P$. tecunumanii as a resistant host [4].

\section{Transcriptome assembly}

Illumina sequencing and subsequent filtering by Novogene produced ca. 530- and ca. 570 million clean read pairs for $P$. tecunumanii (LE) and $P$. patula, respectively (Additional file 1: Table S1). Following read trimming and filtering, the $P$. tecunumanii (LE) full dataset contained a total of ca. 870 million reads (81.6\% of the clean reads) representing ca. $120 \mathrm{~Gb}$ of sequence; the $P$. patula full dataset contained ca. 950 million reads $(83.5 \%$ of clean reads) representing ca. $130 \mathrm{~Gb}$ of sequence. Normalisation to $100 \mathrm{X}$ read coverage retained ca. 54 million reads $(6.2 \%$ of the full dataset) in the normalised dataset for P. tecunumanii (LE) and ca. 77 million reads $(8.1 \%$ of the full dataset) in the normalised dataset for P. patula.

Four de novo assemblies, with two different $k$-mer lengths and stringencies, were constructed using the full dataset (Fig. 2). The normalised dataset was used to construct eight de novo and eight genome guided assemblies; using two different $k$-mer lengths, stringencies and reconstruction algorithms. Preliminary assembly generated 20 transcriptomes containing a total of 3,023,703 transcripts for $P$. tecunumanii (LE) and 5,868,982 transcripts

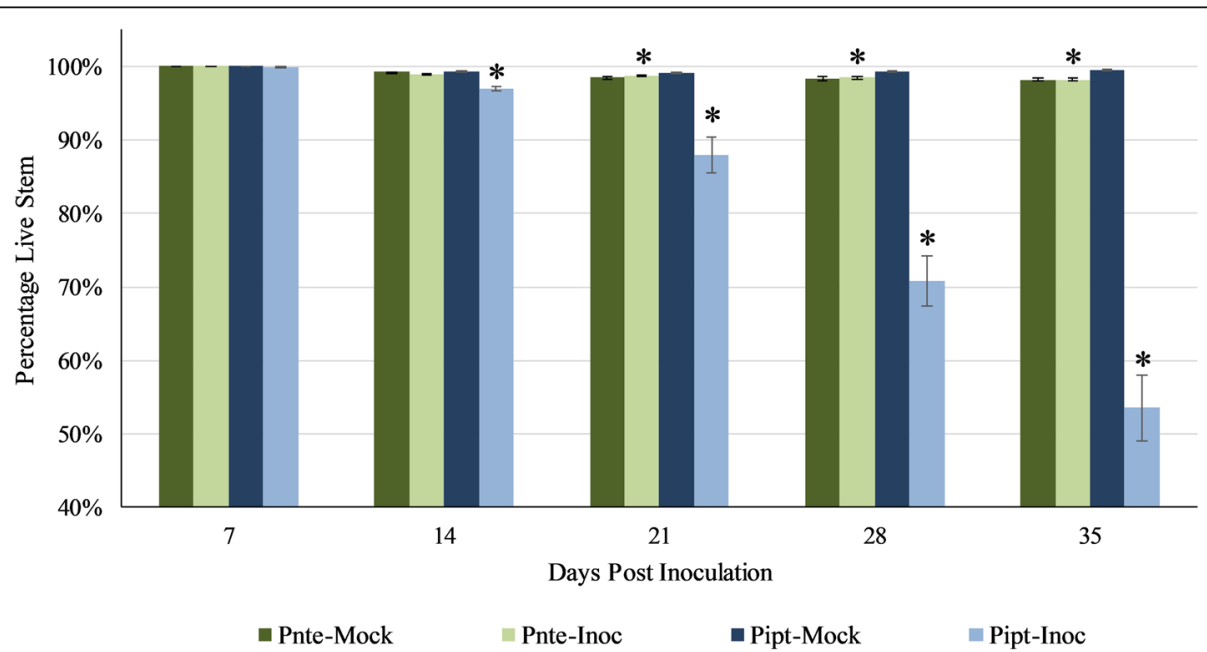

Fig. 1 Fusarium circinatum disease progression on inoculated low elevation Pinus tecunumanii and Pinus patula seedlings. Error bars represent the standard error of the mean (Inoc $n=100 ;$ Mock $n=20$ ). Pnte - P. tecunumanii; Pipt - P. patula; Inoc - inoculated; Mock - Mock-inoculated; * significant difference between inoculated and mock-inoculated groups (Kruskal-Wallis rank sum test, $p<0.05$ ) 


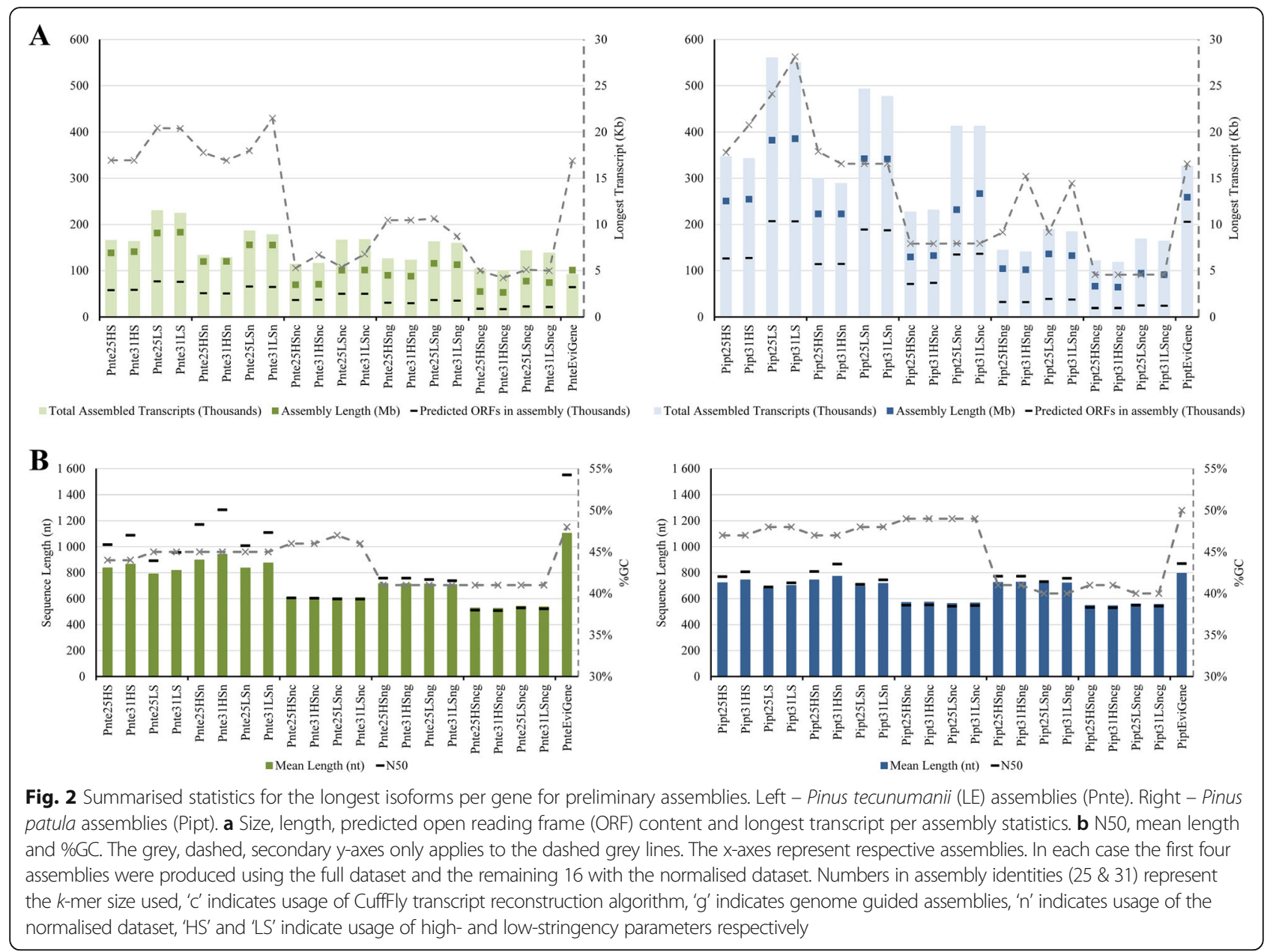

for $P$. patula, after filtering assemblies to only retain the longest isoform per gene (Fig. 2).

Redundancy across the preliminary transcripts was reduced based on coding potential using the tr2aacds pipeline from EvidentialGene. Following open reading frame (ORF) prediction, $36.2 \%$ and $37.4 \%$ of input sequences were classified as non-redundant, for $P$. tecunumanii (LE) and $P$. patula respectively, of which $29.6 \%$ and $37.4 \%$ were retained as informative after substring dereplication using CD-HIT-EST v4.6.1 [48, 49]. Removal of low quality transcripts resulted in 182,681 sequences grouped into 91,552 loci for $P$. tecunumanii (LE), defined as the PnteEviGene assembly, and 542,459 sequences grouped into 325,974 loci for P. patula, defined as the PiptEviGene assembly (Fig. 2). When compared to the $P$. tecunmanii (LE) Trinity assemblies the PnteEviGene assembly showed a better ratio of assembled transcripts to overall assembly length as reflected by its high N50 and mean contig length, which was greater than any of the input assemblies, indicating that the EvidentialGene pipeline successfully selected the longest assembled isoforms from among the Trinity assemblies. For $P$. patula on the other hand, comparison of the EviGene assembly to the Trinity assemblies showed less of an improvement. Still, the PiptEviGene assembly, similar to the PnteEviGene assembly, had a higher average transcript length and N50 than any of the Trinity assemblies.

\section{Annotation}

Expression based filtering of the PnteEviGene and PiptEviGene assemblies retained ca. $85 \%$ and ca. $63 \%$ of transcripts (Table 1). GeneMarkS-T successfully predicted coding regions for ca. 95\% of expressed transcripts for both species, though the $P$. patula assembly contained a ca. $10 \%$ lower proportion of complete reading frames compared to the P. tecunumanii assembly. Protein clustering retained ca. $77 \%$ of the EvidentialGene transcripts for $P$. tecunumanii, while only ca. $51 \%$ of $P$. patula transcripts were retained, indicating that greater redundancy was retained in the P. patula assembly during CD-hit-EST clustering.

Best hit selection of BLAST alignments, $P$. tecunumanii (P. patula) clustered protein sequences, to Arabidopsis, RefSeq and UniProt resulted in informative hits for ca. 
Table 1 Summarised EnTAP annotation statistics

\begin{tabular}{|c|c|c|c|c|}
\hline \multirow{2}{*}{ Assembly Statistics } & \multicolumn{2}{|c|}{ Pinus tecunumanii (LE) } & \multicolumn{2}{|l|}{ Pinus patula } \\
\hline & & & & \\
\hline Assembly & PnteEviGene & Pnte_v1.0 & PiptEviGene & Pipt_v2.0 \\
\hline Total Sequences & 91,552 & 28,621 & 325,974 & 52,735 \\
\hline Total Transcriptome Length (Mb) & 100.95 & 50.01 & 259.02 & 72.15 \\
\hline Average Sequence Length (nt) & 1103 & 1747 & 794 & 1368 \\
\hline N50 (nt) & 1551 & 2296 & 870 & 1897 \\
\hline Longest Sequence (nt) & 16,886 & 16,886 & 16,570 & 16,570 \\
\hline Shortest Sequence (nt) & 351 & 351 & 351 & 351 \\
\hline$\% G C$ & $48 \%$ & $44 \%$ & $50 \%$ & $46 \%$ \\
\hline \multicolumn{5}{|l|}{ Sequence filtering ${ }^{a}$} \\
\hline \multirow[t]{2}{*}{ Sequences with FPKM > 1} & 77,563 & & 203,996 & \\
\hline & $(84.72 \%)$ & & $(62.58 \%)$ & \\
\hline \multirow[t]{2}{*}{ Sequences with GeneMarkS-T predicted CDS } & 74,556 & & 194,568 & \\
\hline & $(81.44 \%)$ & & $(59.69 \%)$ & \\
\hline \multirow[t]{2}{*}{ Total proteins after clustering to $90 \%$ identity } & 70,748 & & 167,961 & \\
\hline & $(77.28 \%)$ & & $(51.53 \%)$ & \\
\hline \multicolumn{5}{|l|}{ Annotation $^{\mathrm{b}}$} \\
\hline & \multicolumn{2}{|c|}{$\begin{array}{l}\text { Predicted protein } \\
\text { frame }\end{array}$} & & \\
\hline \multirow[t]{2}{*}{ Complete } & 32,193 & 18,971 & 61,134 & 26,835 \\
\hline & $(45.50 \%)$ & $(66.27 \%)$ & $(36.40 \%)$ & (50.89\%) \\
\hline \multirow[t]{2}{*}{ Internal } & 18,279 & 3347 & 57,027 & 9602 \\
\hline & $(25.84 \%)$ & (11.70\%) & (33.95\%) & $(18.21 \%)$ \\
\hline \multirow[t]{2}{*}{ 3'-partial } & 6686 & 1895 & 21,848 & 4354 \\
\hline & (9.45\%) & $(6.63 \%)$ & $(13.01 \%)$ & (8.26\%) \\
\hline \multirow[t]{3}{*}{ 5'-partial } & 17,398 & 4408 & 54,559 & 11,944 \\
\hline & (24.59\%) & $(15.40 \%)$ & $(32.48 \%)$ & (22.65\%) \\
\hline & $\begin{array}{l}\text { Similarity Sear } \\
\text { Annotation }\end{array}$ & & & \\
\hline \multirow[t]{2}{*}{ Sequences with informative BLASTp alignments } & 19,296 & 15,192 & 38,714 & 27,328 \\
\hline & $(27.27 \%)$ & (53.09\%) & $(23.05 \%)$ & $(51.82 \%)$ \\
\hline \multirow[t]{2}{*}{ Sequences with uninformative BLASTp alignments } & 15,437 & 6131 & 34,101 & 9261 \\
\hline & $(21.82 \%)$ & (21.41\%) & $(20.30 \%)$ & $(17.56 \%)$ \\
\hline \multirow[t]{3}{*}{ Sequences without BLASTp alignments } & 36,015 & 7300 & 95,146 & 16,147 \\
\hline & (50.91\%) & (25.50\%) & $(56.65 \%)$ & (30.62\%) \\
\hline & $\begin{array}{l}\text { Functional } \\
\text { Annotation }\end{array}$ & & & \\
\hline \multirow[t]{2}{*}{ Sequences with family assignment } & 55,627 & 28,484 & 128,952 & 52,166 \\
\hline & (78.63\%) & (99.52\%) & (76.77\%) & (98.92\%) \\
\hline \multirow[t]{2}{*}{ Sequences with at least one $\mathrm{GO}$ term } & 31,640 & 16,197 & 77,063 & 33,712 \\
\hline & $(44.72 \%)$ & (56.60\%) & $(45.88 \%)$ & (63.93\%) \\
\hline \multirow[t]{3}{*}{ Sequences with at least one pathway (KEGG) assignment } & 17,303 & 8004 & 47,383 & 21,094 \\
\hline & $(24.46 \%)$ & (27.98\%) & $(28.21 \%)$ & $(40.00 \%)$ \\
\hline & $\begin{array}{l}\text { Annotation } \\
\text { Summary }\end{array}$ & & & \\
\hline
\end{tabular}


Table 1 Summarised EnTAP annotation statistics (Continued)

\begin{tabular}{|c|c|c|c|c|}
\hline \multirow[b]{2}{*}{ Unannotated Sequences } & \multicolumn{2}{|c|}{ Pinus tecunumanii (LE) } & \multicolumn{2}{|c|}{ Pinus patula } \\
\hline & 14,568 & 0 & 36,698 & 0 \\
\hline & (20.59\%) & $(0.00 \%)$ & (21.85\%) & $(0.00 \%)$ \\
\hline \multirow[t]{2}{*}{ Total sequences annotated } & 56,180 & 28,621 & 131,263 & 52,735 \\
\hline & (79.41\%) & (100.00\%) & (78.15\%) & $(100.00 \%$ \\
\hline \multirow[t]{2}{*}{ Non-pine origin sequences } & 27,550 & 0 & 78,527 & 0 \\
\hline & (38.94\%) & (0.00\%) & (46.75\%) & (0.00\%) \\
\hline
\end{tabular}

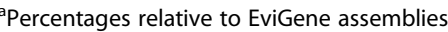

${ }^{b}$ Percentages relative to clustered GeneMarkS-T assemblies for EviGene columns and relative to total sequences for Pnte_v1.0 and Pipt_v2.0

$27 \%$ (23\%) of contigs, uninformative hits for ca. $22 \%$ (20\%) of contigs and no hits for ca. 51\% (57\%) of contigs. EggNOG functional annotation assigned ca. 79\% (77\%) of contigs to orthologous groups. In total, ca. $79 \%$ (78\%) of contigs were successfully annotated. Taxonomy based filtering identified 27,550 $(78,527)$ non-pine origin contigs.

Removal of non-pine origin contigs produced the first $P$. tecunumanii draft transcriptome (Pnte_v1.0), containing 28,621 contigs of which ca. $53 \%$ had informative BLAST annotations and ca. 99\% were assigned to EggNOG functional annotations (Additional file 2: Table S6). The current P. patula draft transcriptome (Pipt_v2.0) contained 52,735 contigs of which ca. $52 \%$ and $99 \%$ had informative BLAST annotations and EggNOG functional annotations respectively (Additional file 3: Table S7), an improvement on the 60\% annotation of Pipt_v1.0 [12]. TAIR identifiers could be assigned to 16,393 (ca. 57\%) Pnte_v2.0 and 27,954 (ca. 53\%) Pipt_v2.0 contigs (Additional file 4: Table S8, Additional file 5: Table S9). Best hit annotation of non-pine origin sequences indicated that the majority of contaminants were of fungal origin (Additional file 1: Table S5).

Gene Ontology (GO) terms were assigned to ca. 57\% $(16,197)$ of $P$. tecunumanii contigs; 11,157 contigs with biological process (BP) terms, 8086 contigs with molecular function (MF) terms, and 15,077 contigs with cellular compartment (CC) terms (Table 1). For P. patula, GO terms were assigned to ca. $64 \%$ (33,712) of contigs; 24,709 contigs with BP terms, 18,956 contigs with MF terms and 31,760 contigs with CC terms. Among the top MF terms identified for both species were hydrolase activity, transferase activity, ion binding, protein binding and two parent terms for nucleic acid binding (organic cyclic acid binding, heterocyclic compound binding), similar to what has been observed for other conifer reference sequences $[11,12,50,51]$ (Fig. 3). The most common BP terms were indicative of rapid and extensive metabolic activity within the analysed tissue, as has been shown for the interaction between $P$. radiata and F. circinatum [11].

Completeness (total complete and fragmented BUSCOs), contiguity (total complete BUSCOs/total complete and fragmented BUSCOs) and redundancy (duplicated
BUSCOs) of the assemblies was determined by comparison to the BUSCO eukaryote $(n=303)$ and embryophyte $(n=1440)$ lineage datasets [42] (Fig. 4). When compared to the eukaryotic lineage the BUSCO results for Pnte_v1.0 and Pipt_v2.0 (C:96.7\%,[S:58.4\%,D:38.3\%],F:1.0\%,M:2.3\% and C:97.7\%,[S:43.6\%,D:54.1\%],F:1.0\%,M:1.3\%; where C refers to the percentage of complete BUSCOs, $\mathrm{S}$ refers to the percentage of complete and single copy BUSCOs, D refers to complete and duplicated BUSCOs, F refers to fragmented BUSCOs and M refers to missing BUSCOs) showed high completeness (97.7\% and $98.7 \%$ ) and contiguity (99.0\% and $99.0 \%$ ) for both assemblies. While redundancy (38.3\% and 54.1\%) was also high for both assemblies, a similar trend was observed for the other gymnosperm assemblies analysed (Fig. 4). Comparison to the embryophyte lineage (Pnte_v1.0 $=\mathrm{C}: 87.0 \%,[\mathrm{~S}: 77.2 \%, \mathrm{D}: 9.9 \%], \mathrm{F}: 2.8 \%, \mathrm{M}: 10.2 \%$ and Pipt_v2.0 = C:87.7\%,[S:76.1\%,D:11.6\%],F:2.6\%,M:9.7\%) showed lower redundancy (9.9\% and $11.6 \%)$. The completeness $(89.8 \%$ and 90.3\%) and contiguity (96.9\% and 97.2\%) of Pnte_v1.0 and Pipt_v2.0 for the embryophyte lineage was the highest amongst analysed assemblies. The high redundancy in the assemblies likely reflects assembled haplotypes [42] due to the high genetic variance present in the populations from which the data was generated, indicating that more variance was present in the $P$. patula seedlings compared to the $P$. tecunumanii (LE) seedlings.

\section{Identification of pathogenesis-related gene families}

A total of 51,594 orthogroups were identified, containing 2,599,567 genes, of which 248 groups contained genes from all 94 species. For Pnte_v1.0, 28,298 (98.8\%) contigs were assigned to 9561 orthogroups, of which 4 , containing 8 contigs, were species specific. For Pipt_v2.0, 50,244 (95.3\%) contigs were assigned to 11,325 orthogroups, of which 85 , containing 225 contigs, were species specific. Of the total orthogroups, 9071 were Gymnosperm specific. A further 7072 were specific to conifers, of which 3181 were specific to pines (Additional file 6: Figure S1).

PR genes identified in $A$. thaliana, B. distachyon, $P$. trichocarpa, O. sativa and V. vinifera [47] were used to identify putative PR orthogroups for 16 of the 17 currently classified plant PR classes, as well as the two 


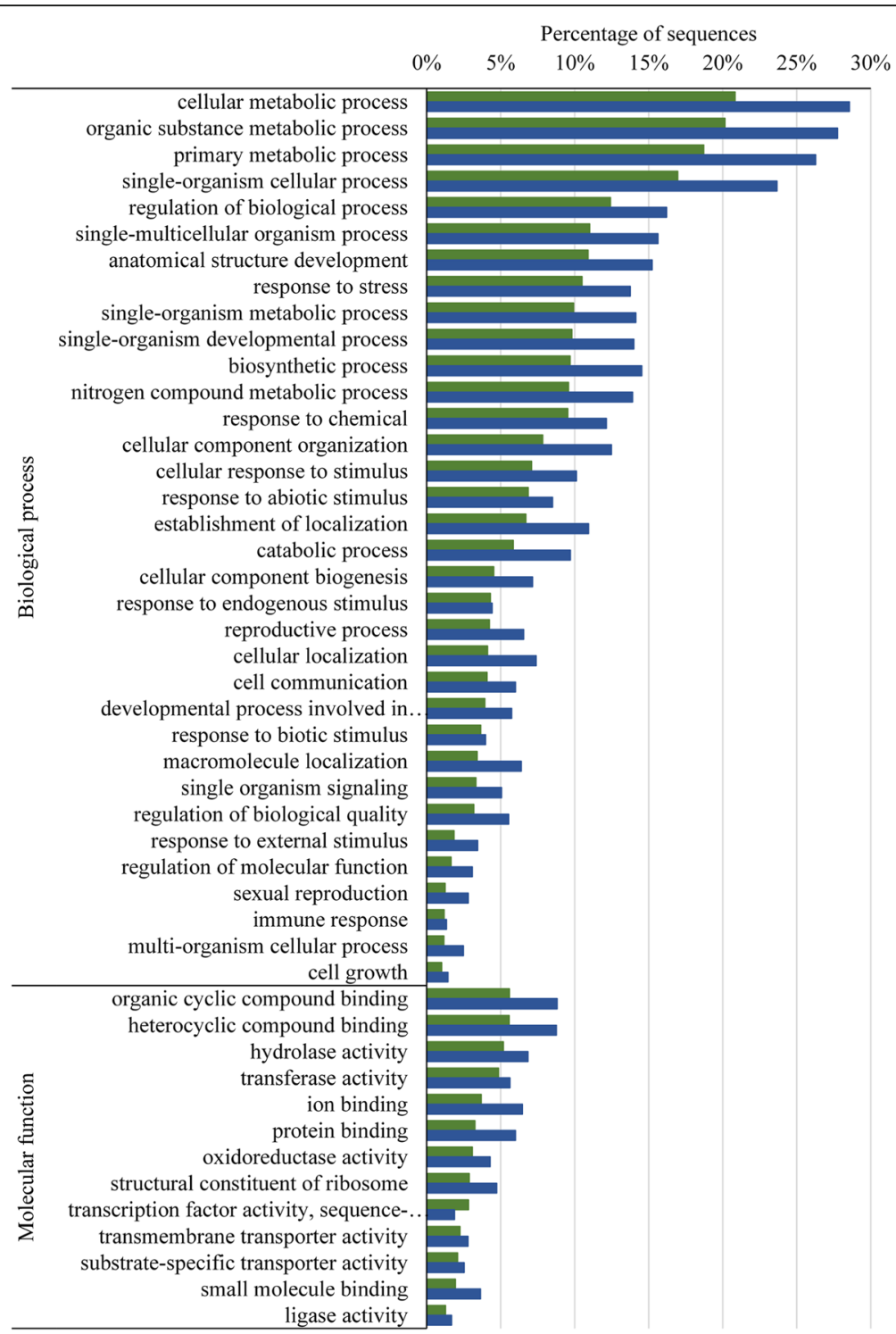

Fig. 3 Distribution of biological process and molecular function Gene Ontology (GO) terms in the assembled transcriptomes. Green - Pinus tecunumanii assembly. Blue - Pinus patula assembly

putative novel classes (Additional file 1: Table S10). PR-15 and PR-16 were both classified as PR-16 due to their high homology and the classification of PR-15 as monocot specific [52]. Putative PR orthogroups were identified for 15 PR families in P. tecunumanii and 16 PR families in P. patula (Additional file 1: Table S11). PR-12 and -13 members were absent from both assemblies. Both of these appear to be angiosperm specific as the PR-12 orthogroup only contained sequences from the basal angiosperm A. trichopoda and dicots, while the PR-13 orthogroup only contained sequences from monocots and brassicaceae, similar to what has previously been observed $[47,53]$. The PR- 6 family was absent from the $P$. tecunumanii assembly, indicative of insufficient expression for assembly rather than absence from the genome. Two putative PR-6 genes were identified in the $P$. patula assembly, interrogation of EggNOG annotations identified the "potato-inhibitor I family domain" found in the PR-6 type member in both sequences.

The only PR classes for which putative members were present in all species were PR-2 and PR-9, although no single orthogroup was present in all species. Putative PR-1 orthologues were only absent from some of the chlorophyte species. PR-7 members were present for all viridiplantae species with PR-8, -11 and -18 , while not identified for all species, similarly present across all viridiplantae lineages. The PR-10 and PR-17 orthogroups only contained sequences from embryophyte species and the PR-14 orthogroup only contained tracheophyte sequences. While some chlorophyte sequences were present, the PR-3, -5 , 

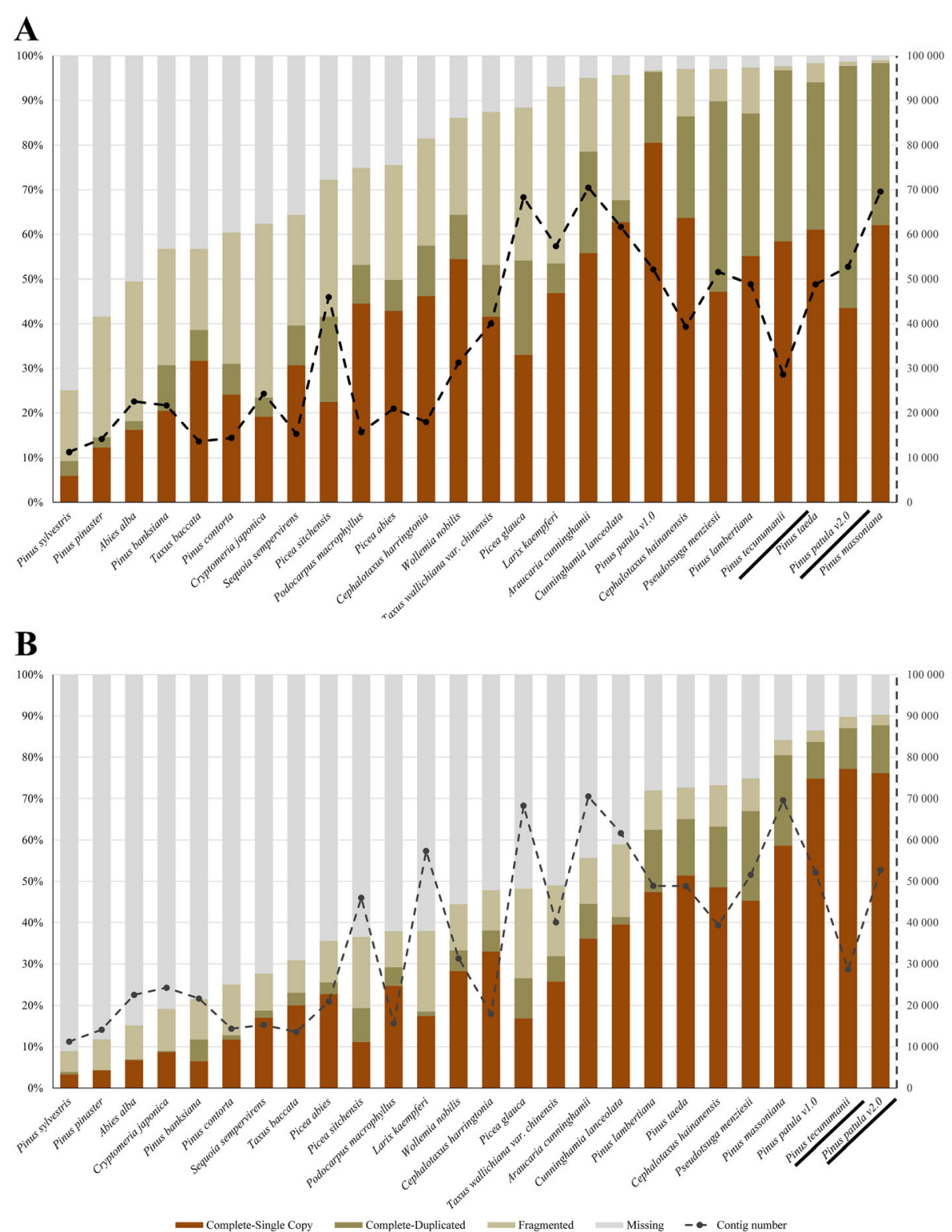

Fig. 4 Comparison of completeness, contiguity and redundancy for assembled transcriptomes (underlined) to other available gymnosperm assemblies. BUSCOs were identified for the (a) eukaryotic $(n=303)$ and $(\mathbf{b})$ embryophyta $(n=1440)$ lineage datasets. The primary $y$-axis refers to the percentage of BUSCOs in each category. The secondary, dashed, $y$-axis refers to the total contig count in each assembly

and -16 orthogroup sequences were mostly spread evenly between the embryophyte lineages. The majority of identified PR-4 and -6 sequences were identified in angiosperms. Despite being initially identified from a dicot, putative PR-19 sequences, as expected, were only identified in the lycophyte $S$. moellendorfii, coniferous gymnosperms, the basal angiosperm A. trichopoda and in low numbers in monocots [54].

Potential lineage specific chitinase gene family expansions were observed when comparing two of the identified PR-3 orthogroups (OG0000134 and OG0000642), as well as the two PR-8 orthogroups identified (Fig. 5).
When looking at the PR-3 orthogroups, both groups appear present in relatively equal amounts in gymnosperms, while OG0000134 is more prominent in angiosperms. The opposite is seen for the two PR-8 groups, with both relatively similar in angiosperms but OG0000252 more prominent in gymnosperms. Interestingly, in both cases the pattern observed for the brassicales is similar to the gymnosperms not the angiosperms.

More putative PR genes were identified in P. patula (801) relative to $P$. tecunumanii (646). While lower amounts of $P$. tecunumanii transcripts in an orthogroup is likely due to the difference in the number of transcripts assembled, the 

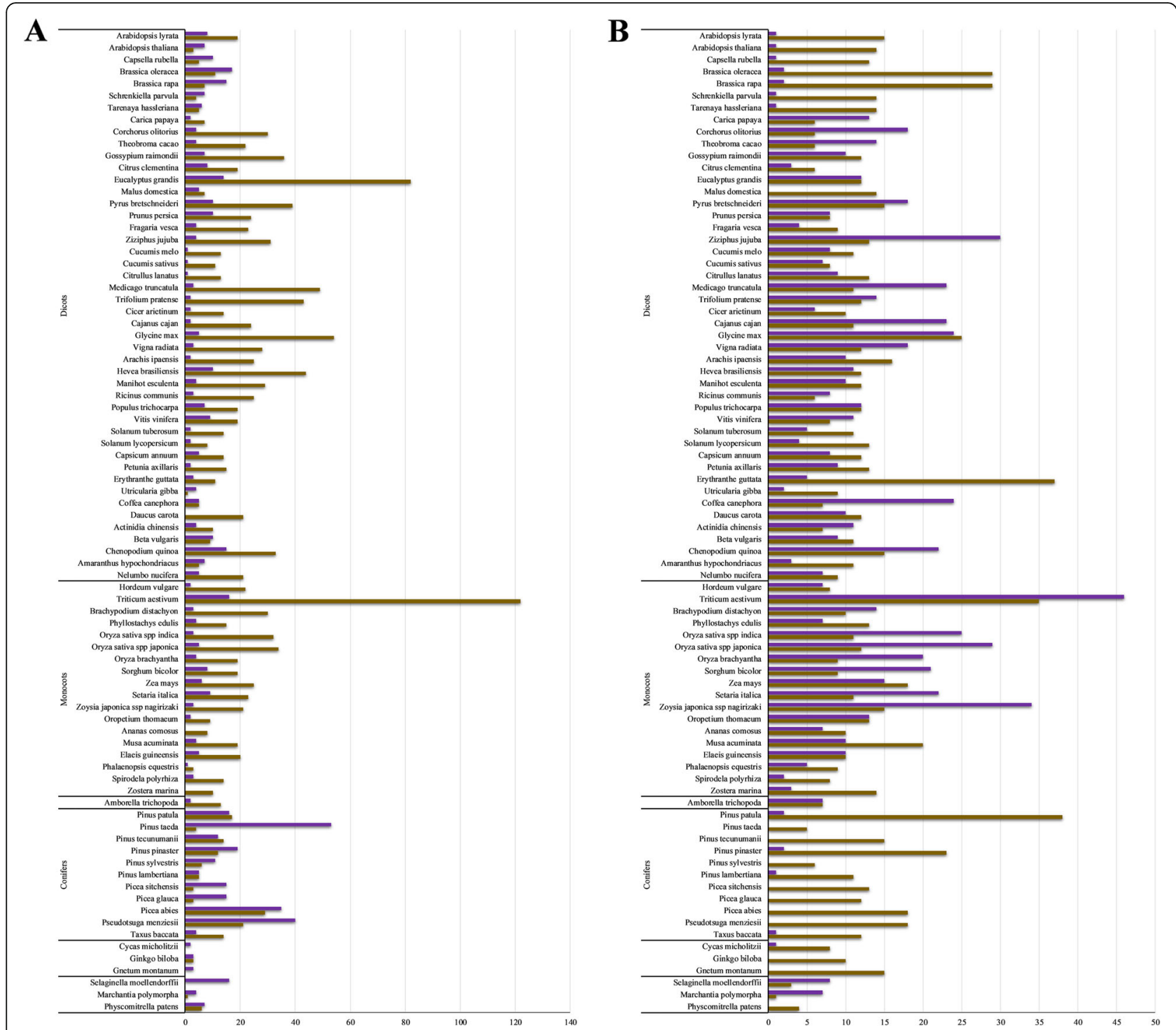

Fig. 5 Comparison of orthogroup size across lineages between two chitinase orthogroups. a PR-3 orthogroups OG0000134 (brown) and OG000642 (purple). b PR-8 orthogroups OG0000252 (brown) and OG0000455 (purple). In both cases the x-axis refers to the amount of proteins per species present in each orthogroup

reverse could indicate transcripts absent from the P. patula defence response. P. patula had more transcripts for all PR gene classes identified except the PR-4 chitinase family (OG0001724), for which 3 transcripts were identified in both species (Fig. 6). The number of transcripts identified for the PR-5, PR-10, PR-11, PR-14, PR-17, PR-18 and PR-19 families, were very similar between species, with at most five transcripts more in $P$. patula. When comparing the four PR-5 orthogroups present in both species, OG0000039 and OG0001011 had the same amount of transcripts and OG0000062 had more $P$. patula transcripts, while OG0000094 had one more $P$. tecunumanii transcript. Of the $51 \mathrm{PR}$ orthogroups containing transcripts from either species, 3 only had P. patula transcripts, 26 had more $P$. patula than $P$. tecunumanii transcripts, 14 had the same amount of transcripts and only 8 had more $P$. tecunumanii transcripts. Similar to the PR-5 orthogroup (OG0000094), two PR-2 (OG0000138; OG0000235) and two PR-9 (OG0000400; OG0002881) orthogroups had one more $P$. tecunumanii transcript. An additional two PR-9 (OG0000025; OG0000107) and one PR-1 (OG0000244) orthogroup had two more transcripts from $P$. tecunumanii compared to $P$. patula. The relative expansion observed in four of the PR-9 peroxidase orthogroups could indicate a more robust cell wall reinforcement or oxidative burst response in $P$. tecunumanii, however, this remains to be functionally determined. 


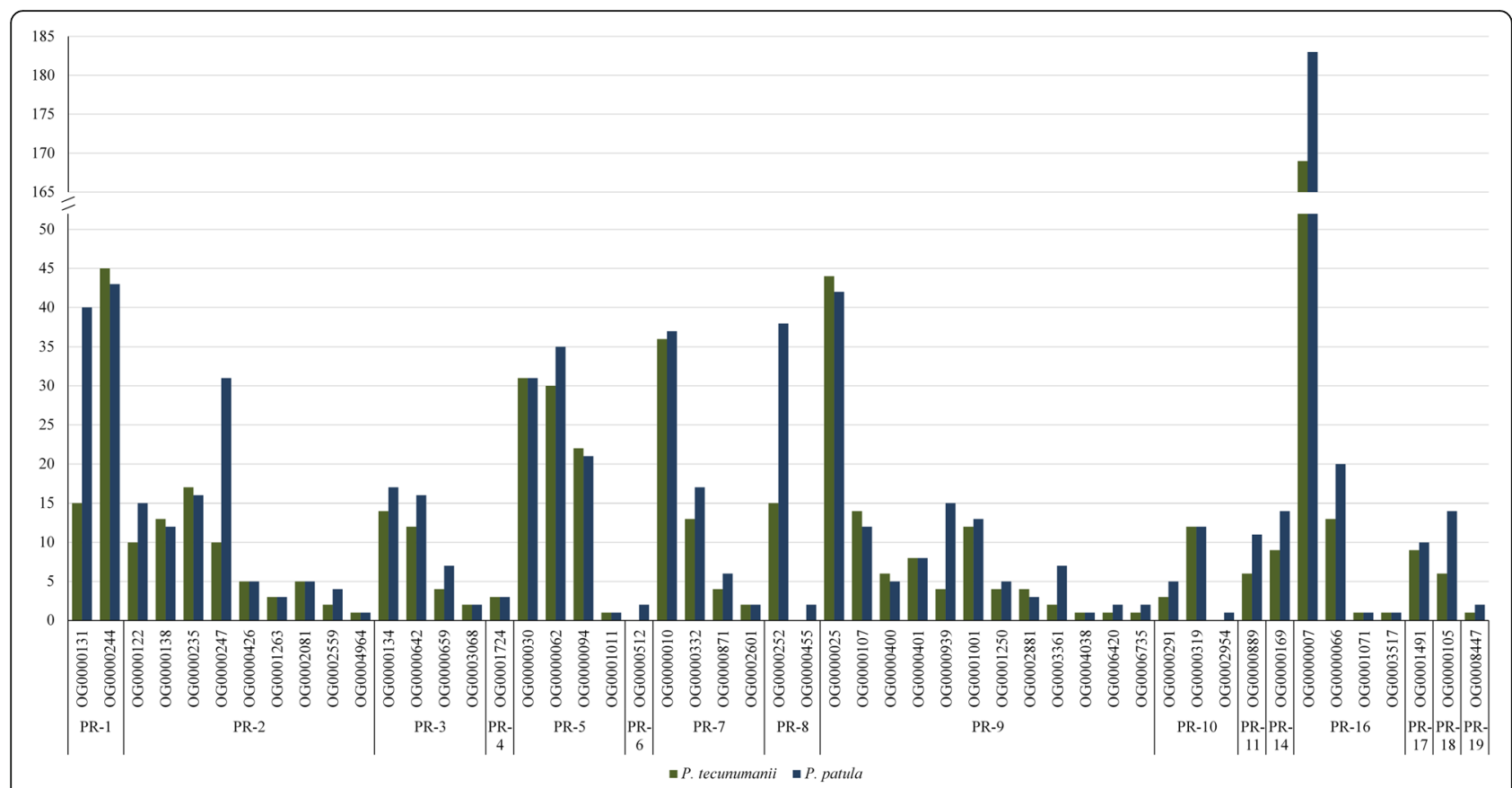

Fig. 6 Putative Pathogenesis Related orthogroup transcript counts in P. tecunumanii and P. patula. The y-axis represents the transcript count for each orthogroup

\section{Conclusions}

In summary, we report the assembled juvenile shoot transcriptome for Pinus tecunumanii, the first reference sequence for this species, as well as a comparable juvenile shoot transcriptome for P. patula. Both assemblies represent important resources that will contribute to further study of the Pinus-F. circinatum interaction. Furthermore, of the 19 PR classes, putative homologues for 15 were identified in $P$. tecunumanii and for 16 in $P$. patula, resulting in a total of 646 and 801 putative PR genes respectively. This work provides a critical base for future investigation of host-pathogen interactions in these tropical pine species as well as characterisation of other, non-defence related molecular pathways. The assembled transcriptomes will be used as reference to investigate host expression during $F$. circinatum challenge, allowing comparison of resistant and susceptible host responses between closely related species. In addition, the transcriptomes could be used to help characterise genetic markers for these tropical pines.

\section{Additional files}

Additional file 1: Table S1. RNA-sequencing library statistics. Table S2. Program parameters used for assembly that deviated from the default. Table S3. BUSCO input transcriptome sources. Table S4. OrthoFinder input proteome sources. Table S5. Best hit species distributions. Table S10. Putative PR gene family counts. Table S11. Putative Pinus tecunumanii and Pinus patula PR genes. (XLSX $110 \mathrm{~kb}$ )

Additional file 2: Table S6. Pnte_v1.0 annotation. (TSV $48464 \mathrm{~kb}$ )
Additional file 3: Table S7. Pipt_v2.0 annotation. (TSV 124607 kb)

Additional file 4: Table S8. Pnte_v1.0 TAIR identifiers. (TSV 2028 kb)

Additional file 5: Table S9. Pipt_V2.0 TAIR identifiers. (TSV $3421 \mathrm{~kb}$ )

Additional file 6: Figure S1. Orthologous group distribution across analysed lineages. Secondary and tertiary Venn diagrams were constructed based on the lineage specific orthogroups. (TIFF $2590 \mathrm{~kb}$ )

\section{Abbreviations}

BLAST: Basic Local Alignment Search Tool; BP: Biological process; BR: Biological replicate; BUSCO: Benchmarking Universal Single Copy Orthologs; CC: Cellular compartment; CDS: Coding DNA sequence; DAMP: Damage Associated Molecular Pattern; DEG: Differentially Expressed Gene; Dpi: Days post inoculation; EnTAP: eukaryotic non-model Transcriptome Annotation Pipeline; FPKM: Fragments Per Kilobase of transcript per Million mapped reads; Gb: Gigabase; GO: Gene Ontology; GSNAP: Genomic Short-read Nucleotide Alignment Program; KEGG: Kyoto Encyclopedia of Genes and Genomes; LE: Low elevation; MAMP: Microbe Associated Molecular Pattern; Mb: Megabase; MF: Molecular function; ORF: Open reading frame;

PAMP: Pathogen Associated Molecular Pattern; PDA: Potato dextrose agar; PR genes: Pathogenesis-Related genes; PRR: Pattern Recognition Receptor; R gene: Resistance gene; RNA-seq: RNA sequencing; ROS: Reactive oxygen species; RSEM: RNA Sequencing by Expectation Maximisation;

SAR: Systemic acquired resistance; TAIR: The Arabidopsis Information Resource

\section{Acknowledgements}

We thank Dr. Kitt Payn from Mondi and Dr. Nicky Jones from Sappi for their assistance in obtaining plant materials and their support. We are grateful to members of the Eucalyptus and Pine Pathogen Interactions Group and Mr. Jako Visser for assistance with inoculation trials.

\section{Funding}

This work was supported by the National Research Foundation (NRF) of South Africa Scarce Skills Doctoral Scholarship Programme (Grant ID 97892), the NRF Bioinformatics and Functional Genomics Programme (Grant IDs 86936 and 97911) and a strategic grant from the Department of Science and Technology (DST) for the Tree Genomics Platform at the University of 
Pretoria. Further support was provided by Sappi, Mondi, York Timbers and Hans Merensky Foundation though the Forest Molecular Genetics (FMG) Programme with co-funding from the Technology and Human Resources for Industry Programme (THRIP, Grant ID 96413). Funding bodies had no involvement with study design, data collection, analysis and interpretation or preparations of this manuscript. Opinions expressed and conclusion arrived at are those of the author(s) and are not necessarily to be attributed to the NRF.

\section{Availability of data and materials}

The datasets supporting the results of this article are available through the NCBI: Pnte_v1.0 - BioProject, [PRJNA416697]; SRA, [SRR6244829 - SRR6244841]; TSA, [GFZL00000000].

Pipt_v2.0 - BioProject, [PRNJA416698]; SRA, [SRR6246498 - SRR6246510]; TSA, [GGAU00000000].

\section{Authors' contributions}

EAV performed the experimental work, data analysis, interpretation and drafted the manuscript. SN conceived the study, obtained funding to support the research, participated in study design and coordination, and assisted in biological interpretation of data and drafting of the manuscript. JLW provided technical support and computational resources for data analysis, participated in study design, and assisted with evaluation of the manuscript. AAM participated in the experimental design of the study and assisted with critical evaluation of the manuscript. All authors have read and approved the final version of the manuscript.

\section{Ethics approval and consent to participate}

Not applicable. Pine seedlings used in this study were sourced from Sappi and Mondi South African commercial breeding programmes. The seedlings were kindly provided by Dr. Nicky Jones, Sappi Shaw Research Centre and Dr. Kitt Payn, Mondi Forests, Trahar Technology Centre. No field permissions were necessary to collect the plant samples.

\section{Consent for publication}

Not applicable.

\section{Competing interests}

The authors declare that they have no competing interests.

\section{Publisher's Note}

Springer Nature remains neutral with regard to jurisdictional claims in published maps and institutional affiliations.

\section{Author details}

'Department of Biochemistry, Genetics and Microbiology, Forestry and Agricultural Biotechnology Institute (FABI), Genomics Research Institute (GRI), University of Pretoria, Private bag X20, Pretoria 0028, South Africa. ${ }^{2}$ Department of Ecology and Evolutionary Biology, University of Connecticut, Storrs, CT 06269, USA.

Received: 3 January 2018 Accepted: 14 August 2018

Published online: 23 August 2018

\section{References}

1. Nirenberg I, O'Donnell K. New Fusarium species and combinations within the Giberella fujikuroi species complex. Mycologia. 1998;90:434-58.

2. Gordon TR, Swett CL, Wingfield MJ. Management of Fusarium diseases affecting conifers. Crop Prot. 2015;73:28-39.

3. Wingfield MJ, Hammerbacher A, Ganley RJ, Steenkamp ET, Gordon TR, Wingfield BD, Coutinho TA. Pitch canker caused by Fusarium circinatum-a growing threat to pine plantations and forests worldwide. Australas Plant Path. 2008;37:319-34.

4. Hodge GR, Dvorak WS. Differential responses of central American and Mexican pine species and Pinus radiata to infection by the pitch canker fungus. New For. 2000;19:241-58.

5. Roux J, Eisenberg B, Kanzler A, Nel A, Coetzee V, Kietzka E, Wingfield MJ. Testing of selected south African Pinus hybrids and families for tolerance to the pitch canker pathogen, Fusarium circinatum. New For. 2007;33:109-23.

6. Mitchell RG, Wingfield MJ, Hodge GR, Steenkamp ET, Coutinho TA. Selection of Pinus spp. in South Africa for tolerance to infection by the pitch canker fungus. New For. 2012;43:473-89.
7. Kanzler A, Nel A, Ford C. Development and commercialisation of the Pinus patula $\times$ P. teucnumanii hybrid in response to the threat of Fusarium circinatum. New For. 2014;45:417-37.

8. Tobias PA, Guest DI. Tree immunity: growing old without an immune system. Trends Plant Sci. 2014;19:367-70.

9. Wang Z, Gerstein M, Snyder M. RNA-Seq: a revolutionary tool for transcriptomics. Nat Rev Genet. 2009;10:57-63.

10. Martin JA, Wang Z. Next-generation transcriptome assembly. Nat Rev Genet 2011;12:671-82.

11. Carrasco A, Wegrzyn JL, Durán R, Fernández M, Donoso A, Rodriguez V, Neale D, Valenzuela S. Expression profiling in Pinus radiata infected with Fusarium circinatum. Tree Genet Genomes. 2017;13:46.

12. Visser EA, Wegrzyn JL, Steenkamp ET, Myburg AA, Naidoo S. Combined de novo and genome guided assembly and annotation of the Pinus patula juvenile shoot transcriptome. BMC Genomics. 2015;16:1057.

13. Macho AP, Zipfel C. Plant PRRs and the activation of innate immune signalling. Mol Cell. 2014;54:263-72

14. Dangl JL, Jones JDG. Plant pathogens and integrated defence responses to infection. Nature. 2004;411:826-33.

15. Jones JDG, Dangl JL. The plant innate immune system. Nature. 2006;444:323-9.

16. Meng X, Zhang S. MAPK cascades in plant disease resistance signaling. Annu Rev Phytopathol. 2013;51:245-66.

17. Pieterse CMJ, Does DV, Zamioudis C, Leon-Reyes A, Wees SCMV. Hormonal modulation of plant immunity. Annu Rev Cell Dev Bi. 2012;28:489-521.

18. O'Brien JA, Daudi A, Butt VS, Bolwell GP. Reactive oxygen species and their role in plant defence and cell wall metabolism. Planta. 2012;236:765-79.

19. Mengiste T. Plant immunity to necrotrophs. Annu Rev Phytopathol. 2012;50: 267-94

20. Robert-Seilaniantz A, Grant M, Jones JDG. Hormone crosstalk in plant disease and defense: more than just jasmonate-salicylate antagonism. Annu Rev Phytopathol. 2011;49:317-43.

21. Fu ZQ, Dong X. Systemic acquired resistance: turning local infection into global defence. Annu Rev Plant Biol. 2013;64:839-63.

22. van Loon LC, van Strien EA. The families of pathogenesis-related proteins, their activities, and comparative analysis of PR-1 type proteins. Physiol Mol Plant Pathol. 1999:55:85-97.

23. van Loon LC, Rep M, Pieterse CMJ. Significance of inducible defense-related proteins in infected plants. Annu Rev Phytopathol. 2006;44:135-62.

24. Gamir J, Darwiche R, van't Hof P, Choudhary V, Stumpe M, Scheniter R, Mauch F. The sterol-binding acitivyt of pathogenesis-related protein 1 reveals the mode of action of an antimicrobial protein. Plant J. 2017;89: 502-9.

25. Passardi F, Penel C, Dunand C. Performing the paradoxical: how plant peroxidases modify the cell wall. Trends Plant Sci. 2004;9:534-40.

26. Custers JHHV, Harrison SJ, Sela-Buurlage MB, Van Deventer E, Lageweg W, et al. Isolation and characterization of a class of carbohydrate oxidases from higher plants, with a role in active defence. Plant J. 2004;39:147-60.

27. Marcus JP, Goulter KC, Green JL, Harrison SJ, Manners JM. Purification of an antimicrobial peptide from Macadamia integrifolia. Eur J Biochem. 1997;244:743-9.

28. Sooriyaarchchi S, Jaber E, Covarrubias AS, Abhayasekera W, Asiegbu FO, Mowbray SL. Expression and $\beta$-glucan binding properties of scots pine (Pinus sylvestris L.) antimicrobial protein (Sp-AMP). Plant Mol Biol. 2011;77:33-45.

29. Andrews S. FastQC a quality control tool for high throughput sequence data. 2012. http://www.bioinformatics.babraham.ac.uk/projects/fastqc/.

30. Grabherr MG, Haas BJ, Yassour M, Levin JZ, Thompson DA, Amit I, Adiconis X, Fan L, Raychowdhury R, Zeng Q, Chen Z, Mauceli E, Hacohen N, Gnirke A, Rhind N, di Palma F, Bruce W, Friedman N, Regev A. Trinity: reconstructing a full-length transcriptome without a genome from RNA-Seq data. Nat Biotechnol. 2011;29:644-52.

31. Bolger AM, Lohse M, Usadel B. Trimmomatic: a flexible trimmer for Illumina sequence data. Bioinformatics. 2014;30:2114-20.

32. Wu TD, Watanabe CK. GMAP: a genomic mapping and alignment program for mRNA and EST sequences. Bioinformatics. 2005;21:1859-75.

33. Gilbert D. Gene-omes built from mRNA seq not genome DNA. In: $7^{\text {th }}$ annual arthropod genomics symposium, Notre Dame; 2013. https://doi.org/10. 7490/f1000research.1112594.1, http://arthropods.eugenes.org/ EvidentialGene/about/EvigeneRNA2013poster.pdf.

34. Smith-Unna RD, Boursnell C, Patro R, Hibberd JM, Kelly S. TransRate: reference free quality assessment of de-novo transcriptome assemblies. Genome Res. 2016;26:1134-44. 
35. Hart AJ, Ginzburg S, Xu MS, Fisher CR, Rahmatpour N, Mitton JB, Paul R, Wegrzyn JL. EnTAP: bringing faster and smarter functional annotation to non-model eukaryotic transcriptomes: bioRxiv; 2018. p. 307868. https://doi. org/10.1101/307868.

36. Langmead B, Salzberg SL. Fast gapped-read alignment with Bowtie2. Nat Methods. 2012;9:357-9.

37. Li B, Dewey CN. RSEM: accurate transcript quantification from RNA-Seq data with or without a reference genome. BMC Bioinformatics. 2011;12:232.

38. Tang S, Lomsadze A, Borodovsky M. GeneMarkS-T: identification of protein coding regions in RNA transcripts. Nucleic Acids Res. 2015;43:e78.

39. Edgar RC. Search and clustering orders of magnitude faster than BLAST. Bioinformatics. 2010;26:2460-1.

40. Buchfink B, Xie C, Huson D. Fast and sensitive protein alignment using diamond. Nat Methods. 2015;12:59-60.

41. Huerta-Cepas J, Szklarczyk D, Forslund K, Cook H, Heller D, Walter MC, Rattei $T$, Mende DR, Sunagawa $S$, Kuhn $M$, Jensen $L$, von Mering C, Bork $P$. Eggnog 4.5: a hierarchical orthology framework with improved functional annotations for eukaryotic, prokaryotic and viral sequences. Nucleic Acids Res. 2016;4:D286-93.

42. Simão FA, Waterhouse RM, loannidis P, Kriventseva EV, Zdobnov EM BUSCO: assessing genome assembly and annotation completeness with single-copy orthologs. Bioinformatics. 2015:31:3210-2.

43. Wegrzyn JL, Lee JM, Tearse BR, Neale DB. TreeGenes: a forest tree genome database. Int J Plant Genomics. 2008;2008:412875.

44. Emms DM, Kelly S. OrthoFinder: solving fundamental biases in whole genome comparisons dramatically inproves orthogroup inference accuracy. Genome Biol. 2015;16:157.

45. Blande D, Halimaa P, Tervahauta Al, Aarts MGM, Kärenlampi SO. De novo transcriptome assemblies of four accessions of the metal hyperaccumulator plant Noccaea caerulescens. Sci Data. 2017;4:160131.

46. Hill CB, Cassin A, Keeble-Gagnère G, Doblin MS, Bacic A, Roessner U. De novo transcriptome assembly and analysis of differentially expressed genes of two barley genotypes reveal root-zone-specific responses to salt exposure. Sci Rep. 2016;6:31558.

47. Fister AS, Mejia LC, Zhang Y, Herre EA, Maximova SN, Guiltinan M. Theobroma cacao . Pathogenesis-related gene tandem array members show diverse expression dynamics in response to pathogen colonization. BMC Genomics. 2016;17:363.

48. Li W, Godzik A. Cd-hit: a fast program for clustering and comparing large sets of protein or nucleotide sequences. Bioinformatics. 2006;22:1658-9.

49. Fu L, Niu B, Zhu Z, Wu S, Li W. CD-HIT: accelerated for clustering the next-generation sequencing data. Bioinformatics. 2012;28:3150-2.

50. Wegrzyn JL, Liechty JD, Stevens KA, Wu LS, Loopstra CA, Vasquez-Gross HA, et al. Unique features of the loblolly pine (Pinus taeda L.) megagenome revealed through sequence annotation. Genetics. 2014;196:891-909.

51. Pavy N, Pelgas B, Laroche J, Rigault P, Isabel N, Bousquet J. A spruce gene map infers ancient plant genome reshuffling and subsequent slow evolution in the gymnosperm lineage leading to extant conifers. BMC Biol. 2012;10:84.

52. Dunwell JM, Gibbings JG, Nahmood T, Saglan Naqvi SM. Germin and germin-like proteins: evolution, structure, and function. Crit Rev Plant Sci. 2008:27:342-75

53. Stec B. Plant thionins - the structural perspective. Cell Mol Life Sci. 2006;63: 1370-85.

54. Manners JM. Primitive Defence: the MiAMP1 antimicrobial peptide family. Plant Mol Biol Rep. 2009;27:237-42.

Ready to submit your research? Choose BMC and benefit from:

- fast, convenient online submission

- thorough peer review by experienced researchers in your field

- rapid publication on acceptance

- support for research data, including large and complex data types

- gold Open Access which fosters wider collaboration and increased citations

- maximum visibility for your research: over $100 \mathrm{M}$ website views per year

At BMC, research is always in progress.

Learn more biomedcentral.com/submissions 